\title{
ON THE HOMOLOGY OF COMPLEMENTS OF ARRANGEMENTS OF SUBSPACES AND SPHERES
}

\author{
YI HU
}

(Communicated by Thomas Goodwillie)

\begin{abstract}
We prove that the Goresky-MacPherson combinatorial formula for the homology of the complement of affine subspaces in Euclidean space $\mathbb{R}^{n}$ holds for much more general "mixed" arrangements of subspaces and spheres.
\end{abstract}

\section{INTRODUCTION AND BASIC DEFINITION}

In this paper we consider the homology groups of the complements of the following mixed arrangements of closed subspaces in $\mathbb{R}^{n}$.

Definition. An arrangement of subspaces and spheres in $\mathbb{R}^{n}$ is a finite set $\mathscr{A}=$ $\left\{A_{0}, \ldots, A_{m}\right\}$ of closed subspaces (with induced topology) of $\mathbb{R}^{n}$, which satisfies

(A0) $\mathscr{A}$ is closed under intersection;

(A1) every $A_{i}$ is a copy of a differentiable ball or sphere of some dimension (that is, of a differentiable copy of $\mathbb{R}^{k}$ or $S^{k}$ for some $k<n$ );

(A2) every two subspaces meet transversally.

This "mixed" case of arrangements contains both the cases of affine subspaces arrangements [GM] and spherical arrangements as special cases (see [ZZ] and [JOS] for the latter arrangements).

Let $M=\mathbb{R}^{n}-\bigcup_{i=0, \ldots, m} A_{i}$ be the complement of the union of these subspaces. In the case that all subspaces $A_{0}, A_{1}, \ldots, A_{m}$ are affine subspaces of Euclidean space $\mathbb{R}^{n}$, there is naturally a partially ordered set (intersection poset) $\mathscr{P}$ attached to the arrangement $\mathscr{A}$ whose elements $v$ correspond to the "flats"

$$
|v|=A_{i_{1}} \cap A_{i_{2}} \cap \cdots \cap A_{i_{r}} .
$$

The poset $\mathscr{P}$ is partially ordered by inclusion, with one maximal element $T$ corresponding to the ambient space $\mathbb{R}^{n}$.

In [GM] Goresky and MacPherson have given-generalizing earlier work of Zaslavsky [Z], Orlik-Solomon [OS], and others-a formula for the homology of the complement $M$.

Received by the editors November 17, 1992.

1991 Mathematics Subject Classification. Primary 57N99. 
Theorem 1.1 [GM]. The homology of the complement of $M=\mathbb{R}^{n}-\bigcup_{i=0, \ldots, m} A_{i}$ is given by

$$
H_{i}(M ; \mathbb{Z}) \cong \bigoplus_{v \in \mathscr{P}} H^{n-d(v)-i-1}\left(K\left(\mathscr{P}_{>v}\right), K\left(\mathscr{P}_{(v, T)}\right) ; \mathbb{Z}\right)
$$

where $K$ is a function sending every poset to its order complex, $d(v)=\operatorname{dim}|v|$, and $H^{-1}(\varnothing, \varnothing)=\mathbb{Z}$ is agreed.

In the case that the collection $\mathscr{A}$ consists of real hyperplanes, this formula reduces to Zaslavsky's formula [Z] for the number of connected components of $M$. If $\mathscr{A}$ is the underlying real arrangement of an arrangement of complex hyperplanes in $\mathbb{C}^{n / 2}$, then this formula reduces to the Orlik-Solomon formula [OS].

Our main aim is to extend the formula to the general mixed arrangements (see §3). In the sequel, we will first give a different proof of the G-M formula (different in the sense that no stratified Morse theory is used). Then as indicated by the different proof, we will be able to give an extension of the G-M formula to our "mixed" arrangements. The main ingredients in our proof are the MayerVietoris sequence, the (half-) links of strata $|v| \subset \mathbb{R}^{n}$, and induction on the size of $\mathscr{P}$.

\section{THE PROOF OF THE G-M FORMULA}

Recall from [GM, Part III, §3.1] that the arrangement $\mathscr{A}$ gives rise to a Whitney stratification of $\mathbb{R}^{n}$, with one stratum $S(v)=|v|-\bigcup_{w<v}|w|$ for each flat $v \in \mathscr{P}$. Furthermore, from [GM, Part III, §3.3 and 3.5], we have the following constructions and statements. Given a flat $v \in \mathscr{P}$, take a point $p \in S(v)$. Let $N$ be an affine subspace of $\mathbb{R}^{n}$ which is complementary to $|v|$ and meets $|v|$ transversely at the point $p$. Also choose $\delta>0$ so small that the closed ball of radius $\delta, B_{\delta}(p)$, intersects only those flats $|w|$ for which $w \geq v$ and such that the boundary $\partial B_{\delta}(p)$ is transverse to the flats $N \cap|w|$. The link $L$ of the point $p$ is the space

$$
L=M \cap N \cap \partial B_{\delta}(p) .
$$

Take a hyperplane in $N$ through $p$ such that $N$ divides $\partial B_{\delta}(p)$ into two semispheres $\partial B_{\delta}^{ \pm}(p)$ and $L^{+}=\partial B_{\delta}^{+}(p) \cap M \cap N$ is homeomorphic to $L^{-}=$ $\partial B_{\delta}^{-}(p) \cap M \cap N . L^{ \pm}$are called half-links. More importantly, by Poincaré duality and [GM, Part III, Corollary 2.3], we have

$$
\begin{aligned}
H_{i}\left(L^{+}, \partial L^{+}\right) & \cong H^{n-d(v)-i-1}\left(\bar{L}^{+}, \bigcup_{i \geq 0} \bar{L}^{+} \cap A_{i}\right) \\
& \cong H^{n-d(v)-i-1}\left(K\left(\mathscr{P}_{>v}\right), K\left(\mathscr{P}_{(v, T)}\right)\right) .
\end{aligned}
$$

We shall use $\mathscr{B}$ to denote the arrangement $\left\{\bar{L}^{+} \cap A_{i}\right\}_{i>0}$.

Now let us denote $H^{n-d(v)-i-1}\left(K\left(\mathscr{P}_{>v}\right), K\left(\mathscr{P}_{(v, T)}\right)\right)$ by $G_{i}^{v}(\mathscr{A})$ and write

$$
G_{i}(\mathscr{A})=\bigoplus_{v \in \mathscr{P}} G_{i}^{v}(\mathscr{A})
$$

We claim that there is a natural homomorphism from $G_{i}(\mathscr{A})$ to $H_{i}(M)$ which can be described as follows. Recall from [GM, Part III, 2.5] there is a natural 
map $G$ (up to homotopy) from $\mathbb{R}^{n}$ to $K(\mathscr{P})$. Then by [GM, Part III, 3.7] we have

$$
\begin{aligned}
G_{i}^{v}(\mathscr{A}) & =H^{n-d(v)-i-1}\left(K\left(\mathscr{P}_{>v}\right), K\left(\mathscr{P}_{(v, T)}\right)\right) \\
& \cong H^{n-d(v)-i-1}\left(K(\mathscr{B}), K\left(\mathscr{B}_{<T}\right)\right) \\
& \stackrel{G^{*}}{\longrightarrow} H^{n-i-d(v)-1}(L, \mathscr{B}) \cong H_{i}(L) .
\end{aligned}
$$

Thus we define $G_{i}^{v}(\mathscr{A}) \rightarrow H_{i}(M)$ to be $G_{i}^{v}(\mathscr{A}) \rightarrow H_{i}(L)$ followed by $H_{i}(L) \rightarrow$ $H_{i}(M)$ induced by inclusion $L \rightarrow M$. Hence we have obtained a natural homomorphism $G_{i}(\mathscr{A}) \rightarrow H_{i}(M)$. Our goal is to show that this homomorphism is an isomorphism.

To achieve this goal, we consider the homology Mayer-Vietoris sequence for the pair

$$
\left(\mathbb{R}^{n}-A_{0}\right)=M_{0} \text { and }\left(\mathbb{R}^{n}-\bigcup_{i \geq 1} A_{i}\right)=M_{1}
$$

and the cohomology Mayer-Vietoris sequence for the pair $\left(\bar{L}^{+}, \bar{L}^{+} \cap A_{0}\right)$, $\left(\bar{L}^{+}, \bigcup_{i \geq 1} \bar{L}^{+} \cap A_{i}\right)$ for every element $v \in \mathscr{P}$. We therefore have

$$
\begin{aligned}
& \rightarrow G_{i+1}\left(\mathscr{A}_{0}\right) \oplus G_{i+1}\left(\mathscr{A}_{1}\right) \rightarrow G_{i+1}\left(\mathscr{A}_{01)} \quad \rightarrow G_{i}(\mathscr{A})\right. \\
& \downarrow \\
& \rightarrow H_{i+1}\left(M_{0}\right) \oplus H_{i+1}\left(M_{1}\right) \rightarrow H_{i+1}\left(M_{0} \cup M_{1}\right) \rightarrow H_{i}\left(M_{0} \cap M_{1}\right) \rightarrow \\
& \rightarrow \quad G_{i}\left(\mathscr{A}_{0}\right) \oplus G_{i}\left(\mathscr{A}_{1}\right) \quad \rightarrow \quad \begin{array}{c}
G_{i}\left(\mathscr{A}_{01}\right) \\
\downarrow
\end{array} \quad \rightarrow \\
& \rightarrow H_{i}\left(M_{0}\right) \oplus H_{i}\left(M_{1}\right) \rightarrow H_{i}\left(M_{0} \cup M_{1}\right) \rightarrow
\end{aligned}
$$

where $\mathscr{A}_{0}$ is the arrangement of $\left\{A_{0}\right\}, \mathscr{A}_{1}$ is the arrangement of $\left\{A_{1}, \ldots, A_{m}\right\}$, and $\mathscr{A}_{01}$ is the arrangement of $\left\{A_{0} \cap A_{1}, \ldots, A_{0} \cap A_{m}\right\}$.

Note that all of the horizontal maps are induced from inclusion and connecting homomorphisms, and all of the vertical maps are induced from Poincaré duality and the map $G$. So the diagram above is cummutative because $G^{*}$ commutes with inclusion-induced maps and connecting homomorphisms. So by induction on the size of the poset $\mathscr{P}$ we have that

$$
G_{*}\left(\mathscr{A}_{0}\right) \oplus G_{*}\left(\mathscr{A}_{1}\right) \rightarrow H_{*}\left(M_{0}\right) \oplus H_{*}\left(M_{1}\right)
$$

and

$$
G_{*}\left(\mathscr{A}_{01}\right) \rightarrow H_{*}\left(M_{01}\right)
$$

are isomorphisms. Hence the homomorphism $G_{*}(\mathscr{A}) \rightarrow H_{*}(M)$ is an isomorphism by the five lemma.

Corollary 2.1. The isomorphism $G_{*}(\mathscr{A}) \rightarrow H_{*}(M)$ is functional with respect to inclusions of subarrangements.

\section{Generalizations}

\subsection{Nonaffine arrangements.}

Definition. A generalized arrangement of subspaces in $\mathbb{R}^{n}$ is a finite set $\mathscr{A}=$ $\left\{A_{0}, \ldots, A_{m}\right\}$ of closed subspaces (with induced topology) of $\mathbb{R}^{n}$, which satisfies

(A0) $\mathscr{A}$ is closed under intersection; 
(A1) every $A_{i}$ is a copy of a differentiable ball of some dimension (that is, of a differentiable copy of $\mathbb{R}^{k}$ for some $k<n$ );

(A2) every two subspaces meet transversally.

We still use $\mathscr{P}$ to denote the intersection poset of $\mathscr{A}$. Then we have the following simple and direct extension of the G-M formula.

Theorem 3.1. The homology of the complement $M=\mathbb{R}^{n}-\bigcup_{i \geq 0} A_{i}$ is given by

$$
H_{i}(M ; \mathbb{Z}) \cong \bigoplus_{v \in \mathscr{P}} H^{n-d(v)-i-1}\left(K\left(\mathscr{P}_{>v}\right), K\left(\mathscr{P}_{(v, T)}\right) ; \mathbb{Z}\right),
$$

and the isomorphism is functorial with respect to inclusions induced by subarrangements.

Proof. Notice that the construction of the link $L$ in the previous section is local. So we can construct the link in the same way except that this time the affine space $N$ is taken to have complementary dimension of $|v|$ and to meet $|v|$ transversally at the chosen point $p$. Thus we still have a natural map $G_{i}(\mathscr{A}) \rightarrow H_{i}(M)$ because the construction of the map $G: \mathbb{R}^{n} \rightarrow K(\mathscr{P})$ does not require the flatness of $|v|, v \in \mathscr{P}$. So the proof in $\S 2$ applies to this generalized arrangement straightforwardly.

3.2. Mixed arrangements of subspaces and spheres. In this section, we shall allow some closed subspaces $A_{i}$ to be spheres.

Definition. An arrangement of subspaces and spheres in $\mathbb{R}^{n}$ is a finite set $\mathscr{A}=$ $\left\{A_{0}, \ldots, A_{m}\right\}$ of closed subspaces (with induced topology) of $\mathbb{R}^{n}$, which satisfies

(A0) $\mathscr{A}$ is closed under intersection;

(A1) every $A_{i}$ is a copy of a differentiable ball or sphere of some dimension (that is, of a differentiable copy of $\mathbb{R}^{k}$ or $S^{k}$ for some $k<n$ );

(A2) every two subspaces meet transversally.

Now we must define a new "intersection" poset $\hat{\mathscr{P}}$ for the arrangement $\mathscr{A}$ because the natural intersection poset $\mathscr{P}$ does not give the correct homology of the complement.

Consider a new arrangement $\hat{A}$ out of $\mathscr{A}$ constructed as follows: If $|v|$ $(v \in \mathscr{P})$ is a differentiable copy of Euclidean space, we retain it. If $|v|$ is a differentiable copy of a sphere, we split it into the disjoint union of two subsets, $p_{v}$ and $|v|-p_{v}$, where $p_{v}$ is a point in $|v|$. But we require that $p_{v}$ satisfies the following compatible property: If $v$ is not a minimal element in $\mathscr{P}$, then $p_{v}=p_{w}$ for some $w<v$. In other words, our operations only apply to the minimal elements $v$ of $\mathscr{P}$ whose underlying subspaces $|v|$ are spheres.

Let $\hat{\mathscr{P}}$ be the intersection poset of $\widehat{\mathscr{A}}$ partially ordered by inclusion. Now we have the following extension of the G-M formula.

Theorem 3.2. The homology of the complement $M=\mathbb{R}^{n}-\bigcup_{i \geq 0} A_{i}$ is given by

$$
H_{i}(M ; \mathbb{Z}) \cong \bigoplus_{v \in \widehat{\mathscr{P}}} H^{n-d(v)-i-1}\left(K\left(\hat{\mathscr{P}}_{>v}\right), K\left(\hat{\mathscr{P}}_{(v, T)}\right) ; \mathbb{Z}\right),
$$

and the isomorphism is functional with respect to inclusions. 
Proof. We still use $|v|$ to denote the underlying space of an element $v \in \hat{\mathscr{P}}$. First note that after the above operation, each $|v|(v \in \hat{\mathscr{P}})$ becomes diffeomorphic to a Euclidean space. This will ensure that our previous arguments apply. Moreover, the compatible property of the points $p_{v}$ will ensure that the formula is true when $\mathscr{A}$ consists of only a copy of a sphere or consists of a copy of a sphere and a copy of a Euclidean space. These two cases must be considered the first step of our induction. To begin the proof, notice again that we can restrict our attention locally. Thus we notice that a small enough neighborhood of a point in an intersection is the same as in the case of $\S 3.1$. So the proof here is the same as in $\S 2$ except that to ensure that the combinatorial formula holds for the first step of the induction we have to split a sphere (if applicable) as a union of a point and its complement in this sphere according to the compatible property.

As indicated by a number of examples in $\mathbb{R}^{3}$, we believe that the transversal condition is a luxury for homology (but is necessary for homotopy; the earlier version of this paper (1990) was in fact an attempt to prove the generalized G-M formula without the transversal condition). Once one can prove this, one probably can tell much more. We also believe that the finite condition imposed on the collection is unnecessary. Instead of imposing the finite condition, we can require a locally finite condition-every intersection $A_{i_{1}} \cap \cdots \cap A_{i_{r}}$ is locally finite. That is, given any point in $\mathbb{R}^{n}$, there is a neighborhood $U$ of it such that the intersection $U \cap A_{i_{1}} \cap \cdots \cap A_{i_{r}}$ has only finitely many connected components. Note that $K(\mathscr{P})$ in this case may be an infinite (locally finite) complex of finite dimension.

\section{ACKNOWLEDGMENTS}

Since the appearance of the basic G-M formula, a number of mathematicians have found that the G-M formula can be proved by more direct combinatorial methods. To my knowledge, they include Ziegler and Zivaljevic [ZZ], Jewell, Orlik, and Shapiro [JOS], Sturmfels, and many others for sure. In particular, Ziegler and Zivaljevic [ZZ] were able to determine homotopy types of the arrangements.

An earlier version (1990) of this paper was collected as an appendix in the author's Ph.D. thesis.

The author thanks Ken Jewell (and Peter Orlik) for pointing out a serious error in the earlier version. He thanks Gunter Ziegler for showing interest in this work and sending him his papers on related subjects. He is very grateful to Robert MacPherson for his valuable encouragement and advice.

\section{REFERENCES}

[GM] M. Goresky and R. MacPherson, Stratified Morse theory, Springer-Verlag, New York, Heidelberg, and Berlin, 1988.

[H] Y. Hu, The geometry and topology of quotient varieties, Ph.D. thesis, MIT, 1991.

[JOS] K. Jewell, P. Orlik, and B. Shapiro, On the complement of affine subspaces arrangements, preprint, University of Wisconsin, 1991.

[OS] P. Orlik and L. Solomon, Combinatorics and topology of complements of hyperplanes, Invent. Math. 56 (1980), 167-189. 
[Z] T. Zaslavsky, Facing up to arrangements: face-count formulas for partitions of space by hyperplanes, Mem. Amer. Math. Soc., vol. 154, Amer. Math. Soc., Providence, RI, 1975.

[ZZ] G. Ziegler and R. Zivaljevic, Homotopy types of subspaces arrangements via diagrams of spaces, preprint, Mittag-Leffler, 1992.

Department of Mathematics, University of Michigan, ANn Arbor, Michigan 48109

E-mail address: yihu@math.lsa.umich.edu 